\title{
Construyendo y adaptando modelos de negocio para la innovación en tiempos de crisis
}

Por. Jaime Daniel Guevara Mira

Máster. Docente investigador del Departamento de

Administración de Empresas UCA·jguevara@uca.edu.sv

\section{Resumen}

En el presente artículo de opinión se hace una breve reseña de la evolución del concepto de modelo de negocio para comprender su importancia y adaptación a los tiempos actuales. De igual manera se presentan los elementos clave para la construcción de modelos de negocio con base en la innovación en tiempos de crisis, tomando en consideración que este concepto es un elemento clave que debe estar centrado en la persona consumidora actual o potencial.

Para la construcción de un modelo de negocio es importante identificar todos los elementos que están sujetos a innovación y que abonan a la generación de propuestas de valor atractivas para el mercado. Para esto se ha tomado como base de análisis el lienzo Canvas propuesto por Osterwalder y Pigneur (2010), donde se plantean nueve factores que se deben analizar y que se encuentran interconectados para la generación de propuestas de valor innovadoras.

\section{Palabras clave:}

Innovación, crisis, modelo de negocio, adaptabilidad, economía, empresa, emprendimiento

\section{Antecedentes del concepto de modelo de negocio}

Para comprender el concepto de modelo de negocio debemos referirnos a la definición inicial realizada por Peter Drucker en 1984, un famoso consultor y profesor de negocios que es considerado el padre de la Administración contemporánea. Éste plantea que un modelo de negocio se refiere a la forma en la que la empresa lleva a cabo su negocio (Drucker, 1985). Pareciera ser un concepto muy simple, pero en el tiempo y contexto en que surgió abrió un panorama que permitió comprender la innovación como un factor que influye puede ser desarrollado en todas las áreas de la empresa, no solamente en el producto o servicio ofrecido al mercado.

El concepto y su base teórica se estudió con profundidad hasta el año 2000, surgiendo así otros autores que buscan explicar la importancia de comprender los modelos de negocio en todos los niveles empresariales. Dentro de estos autores se encuentran Amit y Zoot (2001) que sostienen que un modelo de negocio explica el contenido, la estructura y el gobierno de las transacciones designadas para crear valor al explotar oportunidades de negocio (Amit \& Zott, 2001). 
A continuación, se realiza un breve análisis de los aspectos que se deben considerar al momento de construir o adaptar modelos de negocio actuales, tomando como base la propuesta de Osterwalder y Pigneur (2010) en el modelo del Lienzo Canvas (Figura 1) y agrupando nueve factores: (1) propuesta de valor, (2) segmentación, (3) relación con el cliente (4) canales de entrega de la propuesta de valor, (5) aliados clave, (6) actividades clave, (7) recursos clave, (8) ingresos y (9) egresos, en cuatro preguntas clave: ¿Qué? ¿A quién?, ¿Cómo? y ¿Cuánto?

Figura 1: Lienzo Canvas con preguntas clave

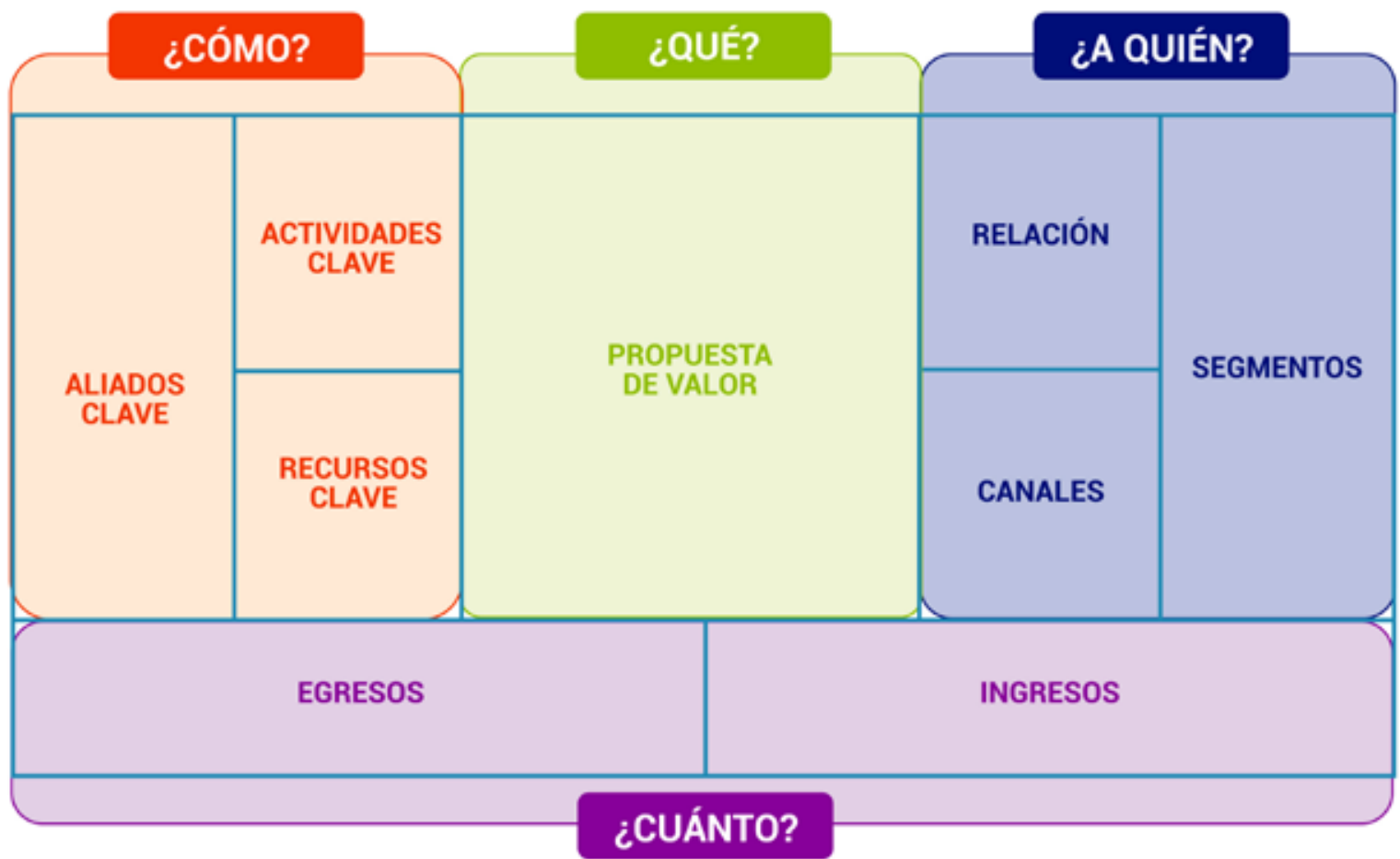

Fuente: Adaptación propia con base en el Lienzo Canvas de Business Model Generation (Osterwalder y Pigneur, 2010)

\section{Segmentación estratégica ¿A quién?}

En primer lugar, es fundamental contar con una descripción detallada del segmento o los segmentos a los que se está ofreciendo la propuesta de valor, de manera que sea el mismo mercado que brinde información relevante para la adaptación oportuna de los productos y/o servicios ofrecidos.

Para el análisis del segmento de manera adecuada se propone comprender el concepto de Adoptador Temprano (Early Adopter), definido por Rogers (1962) a través de la Curva de Adopción de la Innovación (Figura 2). El Early Adopter representa el segmento del mercado (aproximadamente el 13.5\%) que está dispuesto a adoptar los nuevos productos y servicios o los cambios y adaptaciones a la propuesta de valor actual sin ningún cuestionamiento y que, al mismo tiempo, se consideran cocreadores. De manera que están dispuestos a brindar información relevante para generar propuestas de valor con un alto potencial de aceptación por parte del mercado (Rogers, 1962).

De igual manera, los Early Adopters son quienes generan una fuerte influencia en el siguiente segmento, según la curva de innovación, denominado Mayoría Precoz (Early Majority) que representa aproximadamente el $34 \%$ del mercado, un segmento que requiere de referencias ofrecidas por los Early Adopter para superar el abismo que los separa.

Aunque la curva se relaciona directamente con rubros de tecnología se puede realizar un análisis desde todas las perspectivas de negocio, productos $\mathrm{y} / \mathrm{o}$ servicios haciendo una analogía a su ciclo de vida con sus etapas de (1) introducción, (2) crecimiento, (3) madurez y (4) declive. 


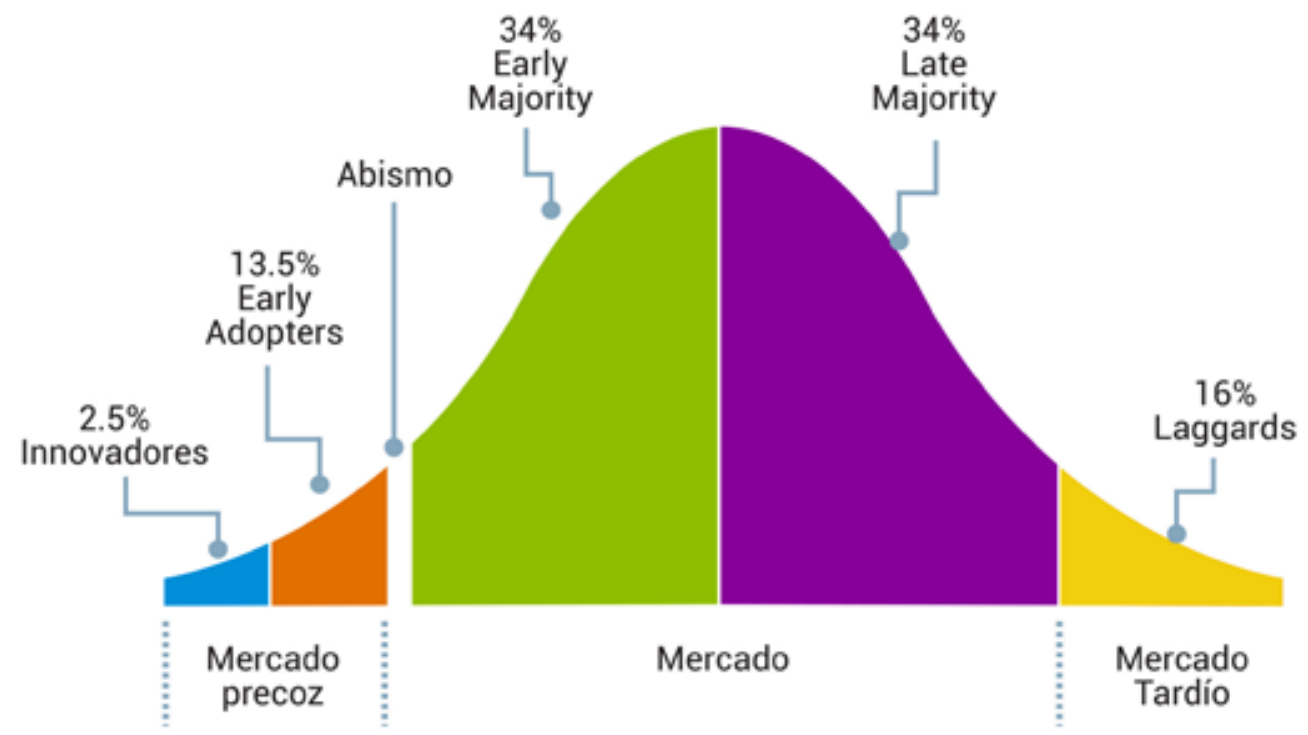

Fuente: Adaptación a partir de la Curva de adopción de la innovación de Rogers (1962).

Es por esta razón que la identificación de los Early Adopter, a partir de datos tanto demográficos como psicográficos, son relevantes para la construcción de propuestas de valor innovadoras. Los datos demográficos brindan información sectorizada a partir de estadísticas de población que nos permite identificar comportamientos que se podrían asociar a ciertos grupos poblacionales tales como edad, nivel socio económico, entre otros. Es importante acompañar este análisis con datos psicográficos, relacionados a gustos, preferencias y estilos de vida, de esta manera podremos tener una mayor comprensión de los objetivos, problemas y barreras del mercado potencial, creando así una propuesta de valor a partir de una realidad palpable, conectando con las necesidades, no solo a nivel funcional, sino que también con las necesidad psicológicas y emocionales del mercado.

\section{Una propuesta de valor innovadora ¿Qué?}

La identificación del segmento al que se desea orientar la propuesta de valor (¿A quién?) brinda la información necesaria para la adaptación continua de la propuesta de valor. La revisión constante del ¿Qué? requiere de una consulta continua y de las condiciones de co-creación junto a nuestro segmento identificado.
La innovación en el ¿Qué? (producto y/o servicio) debe ir acompañada de la construcción de una relación sólida con el segmento. Esta relación se construye mediante la comprensión de la realidad y los beneficios ofrecidos como parte fundamental de la propuesta única de valor, es decir, aquellos beneficios que nadie más ofrece en el mercado actual.

Otro elemento fundamental para la construcción del ¿Qué? es la identificación de los canales de entrega de la propuesta de valor adaptados al segmento. Es tan importante el tipo de relación creado como el cumplimiento de la promesa de venta mediante canales efectivos y valorados por el cliente.

\section{Actividad, recursos y aliados ¿Cómo?}

La innovación también tiene que ver con los procesos relacionados a las actividades y recursos clave, por eso, es fundamental identificar aquellos elementos que abonan a la construcción de la propuesta de valor de manera efectiva. La identificación de recursos y actividades permitirá invertir constantemente en la mejora de los procesos internos y externos mejorando la experiencia del consumidor. Si estos elementos no se identifican de manera clara se pueden generar sobre esfuerzos y fugas de recursos innecesarios, frenando el crecimiento constante del negocio. 
En muchas ocasiones las empresas y emprendimientos no consideran a los aliados como parte fundamental para el fortalecimiento y construcción de la propuesta de valor, que también son parte de los recursos y actividades clave. Dentro de estos aliados se encuentran los proveedores que pueden aportar muchos elementos mediante alianzas o negociaciones de ganar-ganar a través de acuerdos que permitan un fortalecimiento de la cadena de valor. De igual manera existen otros aliados que pueden sumarse como co-creadores o alianzas comerciales para llegar a un mercado más amplio.

\section{Ingresos y egresos ¿Cuánto?}

Finalmente, el ¿Cuánto? permite realizar un planteamiento de innovación para la apertura de nuevas líneas de producto desde la generación de nuevos ingresos. Es decir, la apertura de líneas de producto y/o servicios identificadas como diferentes oportunidades de negocio que van surgiendo en la actividad económica. Para la identificación de estos nuevos ingresos es fundamental considerar los procesos y procedimientos internos e intermedios, buscando oportunidades de mejora continua y que generan un impacto positivo en la percepción del negocio como, por ejemplo, procesos de tratamiento de materiales reciclables, identificación de utilización de materiales intermedios que se van desechando en procesos de fabricación y comercialización, entre otros.

Para la reducción de costos financieros se debe hacer un análisis que influya positivamente en la percepción del mercado frente a la propuesta de valor. Dentro de estos elementos es importante la identificación de los costos más representativos de la empresa, de manera que la innovación permita la adecuación e inversión constante en el control de los costos y el mejor manejo de las finanzas que impactarán directamente en la propuesta de valor.

\section{En conclusión}

La identificación clara de los elementos del modelo de negocio permite tener claridad de todos los esfuerzos que se realizan para generar valor para el mercado actual y potencial. En este sentido, aquellos negocios que logran identificar los elementos que suman valor a la propuesta ofrecida tendrán mayor capacidad para generar cambios o adaptaciones frente a las diferentes crisis que se presenten. En ese sentido, la sistematización del modelo de negocio debe ser un proceso relevante pues permitirá estar preparados para hacer los cambios necesarios de manera efectiva frente a la crisis.

Es importante reconocer que las crisis siempre han existido, a nivel individual, local, regional o global y que, independientemente del nivel de afectación, la capacidad de adaptación depende del mismo negocio y de la flexibilidad en su estructura de innovación.

Es inevitable no mencionar la forma en que la crisis sanitaria mundial generada a partir del COVID 19 ha venido a afectar el funcionamiento y dinámica de muchos negocios a nivel mundial, teniendo que tomar decisiones de manera apresurada sin tener un conocimiento real de su estructura de modelo de negocio.

Esta falta de conocimiento del modelo de negocio ha llevado a muchos emprendimientos a hacer cambios sin tener información oportuna de su mercado actual y los cambios de comportamiento de sus consumidores. Estos cambios generan un riesgo alto para los emprendedores debido a que los recursos son más escasos en la crisis y se debe ser más efectivo en los ajustes que se realicen al negocio, de manera que se obtengan resultados a corto plazo que permitan palear el impacto negativo que generan las crisis.

Finalmente, se puede afirmar que una sistematización del modelo de negocio permitirá que se tomen decisiones acertadas sin impactar negativamente en la propuesta de valor; esta sistematización permitirá que el mismo modelo de negocio sea capaz de transformar las crisis en oportunidades, mediante la identificación de adaptaciones que permitirán aprovechar las nuevas oportunidades que se generan. Es por esta razón que el principal reto de los emprendedores y sus negocios en la actualidad se centra en la comprensión detallada de sus modelos de negocio para llevar a cabo acciones estratégicas que permitan no solo sobrevivir a la crisis, sino descubrir oportunidades de crecimiento constante.

\section{Bibliografia:}

Osterwalder, A. y Pigneur, Y. (2010). Business Model Generation. Centro Libros PAPF, S. L. U. Amit, R. y Zott, C. (2001). Value creation in e business. Strategic Management Journal (22), 493-520. doi:10.1002/smj.187 Drucker, P. F. (1985). Innovation and Entrepreneurship. Routledge Classics. Rogers, E. M. (1962). Diffusion of Innovations. Simon \& Schuster 